\title{
La relación entre el desarrollo de los municipios y la puntuación en Matemáticas: un caso aplicado para Colombia
}

\author{
MORA RODRÍGUEZ, JHON JAMES \\ Universidad Icesi (Colombia) \\ Correo electrónico: jjmora@icesi.edu.co \\ ESTRADA NATES, DANIELA \\ Universidad Icesi (Colombia) \\ Correo electrónico: destrada@icesi.edu.co
}

\begin{abstract}
RESUMEN
Este artículo analiza el efecto del municipio sobre el resultado académico del estudiante de la prueba Estatal Colombiana Saber 11. A partir de modelos lineales jerárquicos con regresión cuantílica nuestros resultados sugieren que el entorno de desarrollo tiene un efecto significativo sobre la punuación en matemáticas para los estudiantes en los cuartiles analizados. De esta forma, los hacedores de política pública deberán tener en cuenta las condiciones del lugar donde viven los estudiantes a la hora de formular políticas educativas encaminadas a mejorar la calidad educativa, ya que esto puede incidir sobre la desigualdad y las oportunidades para acceder a la educación superior.
\end{abstract}

Palabras clave: pruebas Saber 11, regresiones multinivel cuantílicas, desempeño en matemáticas.

Clasificación JEL: A22; C31.

MSC2010: 9711; 9108. 


\title{
The relationship between the municipality development and mathematic score: the Colombian case
}

\begin{abstract}
This article analyzes the effect of the municipality on the academic result of the Colombian State test student "Saber 11". From linear hierarchical models with quantile regression, our results suggest that the type of municipality has a significant effect on students' academic achievement in mathematics for the quartiles studied. In this way, public policy makers must take into account the conditions of the place where students live when formulating educational policies aimed at improving educational quality, since this may affect inequality and opportunities to access higher education.
\end{abstract}

Keywords: Saber 11 test, Multilevel quantile regressions, Mathematic score.

JEL classification: A22; C31.

MSC2010: 9711; 9108.

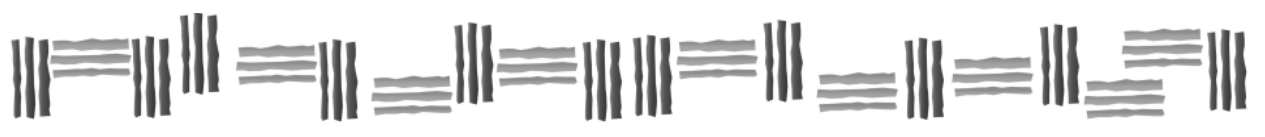




\section{Introducción.}

La brecha en el desarrollo de los municipios contribuye a una inequidad en la distribución de las dotaciones educativas (número de colegios y número de maestros) a nivel regional (Bonet, 2005) y la concentración regional de maestros con altos niveles de calificación (Galvis \& Bonilla, 2012). Es por esta razón, que es importante analizar cómo el grado de desarrollo de un municipio puede afectar sus resultados académicos. Además, como consecuencia de bajos resultados académicos el individuo podría enfrentar restricciones en mercados como el laboral. Por ejemplo, Hanushek (2013) ha encontrado que aquellos que tienen bajo rendimiento académico, tienen más dificultades para participar en el mercado laboral y una mayor probabilidad de tener dificultades para beneficiarse de nuevas oportunidades educativas formales porque carecen de las habilidades para entender problemas complejos, utilizar fórmulas matemáticas y aplicar esos conocimientos en su vida diaria. Cabe observar que la calidad de la educación tiene efectos a nivel microeconómico y macroeconómico, ya que impacta la probabilidad de ingresar a la educación superior (Jiménez et al., 1991), es uno de los determinantes de los ingresos y su distribución (Hanushek \& Woessmann, 2008); además que tiene una correlación significativa con el crecimiento económico y la disminución de la pobreza (Delgado et al., 2014; Hanushek y Woessmann, 2008; Cooray, 2009; Van der Berg, 2008). La calidad de la educación es un concepto amplio; por ejemplo, Rodríguez (1994) propone cuatro categorías para interpretar la calidad: cobertura, gestión pedagógica, resultados y gestión administrativa.

Ahora bien, con el fin de analizar cómo los resultados académicos están relacionados con el nivel de desarrollo económico, utilizamos los resultados de las pruebas Saber 11 en el componente de matemáticas. Esta selección se realizó debido a que la mayoría de los estudiantes próximos a graduarse de secundaria presentan bajos niveles de rendimiento en esta competencia. Además, los resultados en matemáticas de las pruebas del Programa Internacional para la Evaluación de los Estudiantes (o prueba PISA, por sus siglas en inglés) ubicaron a Colombia en el puesto 62 entre 65 países y tres de cada cuatro estudiantes que la tomaron fueron calificados en la categoría de bajo rendimiento (OECD, 2014). Por otro lado, el Departamento de Educación y Empleo de Londres (Department for Education and Employment, 1999) encuentra que una buena competencia en matemáticas tiene un efecto positivo en la productividad promedio de la fuerza laboral y que ésta explica una proporción significativa de las diferencias en el desempeño económico que hay entre los países.

La principal contribución de este artículo consiste en analizar cómo los resultados del componente de matemáticas de la prueba Saber 11 para el año 2014 se ven influenciados por el entorno de desarrollo y la tipología municipal propuestas por el Departamento Nacional de Planeación (DNP), a través de la implementación de un modelo lineal jerárquico por cuartiles. De esta forma, buscamos analizar si el entorno de desarrollo municipal se encuentra relacionado con los resultados en matemáticas (Saber 11) para los cuartiles $0,25,0,5$ y 0,75 cuando se controla por las características individuales y familiares del estudiante y del colegio. El análisis, sobre todo entre los cuartiles $0,25 \mathrm{y}$ 0,75 nos permitirá comparar aquellos estudiantes con resultados bajos versus aquellos estudiantes con resultados altos. De esta forma, en este artículo se evalúa si las tipologías municipales (que conforma los entornos de desarrollo) ayudan a explicar las variaciones en la puntuación en matemáticas para cada uno de los cuartiles analizados. Por último, y no menos importante, nosotros comparamos los resultados obtenidos utilizando una regresión cuantílica y un modelo jerárquico lineal, de manera independiente. La principal razón por la que incluimos el modelo lineal jerárquico por cuantiles consiste en que el colegio explica el $64 \%$ de la variación en los resultados de la puntuación en matemáticas de los estudiantes que se encuentran en la cola inferior de la distribución y que esta variación se incrementa a lo largo de la distribución de las puntuaciones en matemáticas.

Este artículo contiene además de la introducción, cinco secciones adicionales. En la siguiente se describen los factores que pueden afectar el desempeño en matemáticas y la evidencia empírica a nivel internacional y nacional que se ha encontrado al respecto. En la tercera sección se explica la aproximación empírica. Posteriormente, se caracterizan los datos empleados y se presentan las estadísticas descriptivas. En la quinta sección se encuentran los resultados de estimar el modelo 
jerárquico lineal por cuartiles y su respectivo análisis. En la última sección se presentan las conclusiones.

\section{Revisión de la literatura.}

A continuación, se presentan los diferentes modelos que se pueden emplear para responder la pregunta planteada, así como ejemplos de los hallazgos que se han encontrado en el ámbito internacional y nacional sobre los factores que pueden afectar el rendimiento en matemáticas de los estudiantes.

Hoerandner y Lemke (2006), Hanushek (1986) y Hanushek, Machin y Woessmann (2016) utilizan funciones de producción de educación para indagar qué factores están relacionados con los rendimientos en las pruebas estandarizadas. Los resultados pueden variar dependiendo del contexto que se analice, de la riqueza de la base de datos empleada para caracterizar a los colegios e individuos y si el estudio se enfoca en los resultados del estudiante promedio o en la distribución de puntuaciones obtenidas por los estudiantes.

En relación a los resultados individuales, algunos estudios han encontrado que los hombres tienden a obtener mejores resultados que las mujeres (Demir et al., 2010). Haile y Nguyen (2008) concluyen que los individuos afroamericanos e hispanos tienen menores rendimientos en matemáticas, ciencias y lectura que los individuos blancos, aplicando una regresión cuantílica a los datos del Estudio Longitudinal Nacional de Educación del 2000.

Con respecto a los resultados familiares, Bassett et al. (2002) encuentra que los estudiantes de las familias más pobres tienen un peor desempeño en la prueba universitaria americana que aquellos que provienen de una familia acaudalada. Chiu y Xihua (2008) encuentra algo similar para 41 países, pero empleando un índice creado a partir de los años de educación de la madre y el padre y el estatus laboral más importante entre los dos. De igual forma, hay estudios que controlan por el nivel educativo de los padres (Bassett et al., 2002; Haile \& Nguyen, 2008), ocupación de los padres (Haile \& Nguyen, 2008), número de hermanos (Chiu \& Xihua, 2008), entre otras.

Con respecto al colegio, Bassett et al. (2002) encontró que el porcentaje de maestros con maestría y la razón profesor estudiante son significativas y tienen una relación positiva con las puntuaciones en la prueba ACT lo largo de la distribución. Eide y Showalter (1998) no encuentran que estas variables sean significativas empleando la base de datos nacional y longitudinal HS\&B (High School y Beyond). Hanushek (2002) investiga la relación entre el tamaño de las clases y las variables de rendimiento de los estudiantes. Los resultados son mixtos. De los 277 estudios que revisa, informa que el 14\% muestran que la relación entre el tamaño de la clase y el rendimiento de los estudiantes es significativo y positivo, mientras que el 14\% muestran que la relación es significativa, pero negativa, y el $72 \%$ de los estudios encuentra una relación insignificante.

Lubienski y Lubienski (2006) al analizar los resultados de matemáticas de la Evaluación Nacional del Progreso Educativo encuentran que, si no se controla por las características personales y familiares de los individuos, los estudiantes que asisten a colegios privados obtienen en promedio puntuaciones mayores que aquellos que asisten a colegios públicos. Sin embargo, una vez se controla por esas características el efecto desaparece. Jiménez et al. (1991) hallan para Colombia, República Dominicana, Filipinas, Tanzania y Tailandia que incluso si se controla por el ingreso del jefe del hogar, el nivel educativo de los padres y la ocupación de éstos, los estudiantes que asisten a colegios privados obtienen un mejor desempeño que los que asisten a colegios públicos.

Lavy (2015) utilizando los datos de PISA para 2006 (50 países) encuentra evidencia de que el tiempo de instrucción está positiva y significativamente relacionada con las calificaciones de los exámenes, tanto en los países desarrollados como los países en desarrollo. Rivkin y Schiman (2015) 
realizan un estudio similar empleando los resultados de la prueba PISA 2009 (72 países) y encuentran que cuanto mayor sea la duración de la jornada escolar mejor es el rendimiento de los estudiantes.

Con respecto al lugar de residencia, se encuentran los trabajos de Chiu y Xihua (2008) y Fryer y Levitt (2010). El primero incluye el PIB per cápita, el índice de Gini y el grado en que la cultura es individualista para caracterizar a 41 países. Los resultados evidencian que los valores culturales no implican el desempeño en matemáticas; sin embargo, los países más inequitativos en términos de ingresos obtienen puntuaciones menores, de igual forma que aquellos que son más pobres. Por su parte, el segundo artículo incluye un índice de inequidad por género y encuentra que las brechas son casi nulas en países con grandes grados de inequidad por género.

Los análisis realizados para Colombia muestran que el género y la edad son las variables más utilizadas dentro del grupo de factores personales. Estas variables tienden a presentar el mismo signo, el cual indica que los hombres en promedio obtienen puntuaciones en matemáticas superiores a las mujeres, y que cuanto mayor es la edad del estudiante menor es su puntuación (Tabla 1).

Tabla 1. Factores personales y familiares asociados a la puntuación en matemáticas.

\begin{tabular}{|c|c|c|c|c|c|c|}
\hline \multirow[b]{2}{*}{ Autor } & \multirow[b]{2}{*}{ Datos } & \multirow[b]{2}{*}{ Modelo } & \multicolumn{4}{|c|}{ Factores } \\
\hline & & & Hombre & Edad & $\begin{array}{l}\text { Condiciones } \\
\text { económicas }\end{array}$ & Nivel educativo padres \\
\hline $\begin{array}{c}\text { Piñeros \& } \\
\text { Rodríguez (1998) }\end{array}$ & $\begin{array}{c}\text { Saber } 11-1997 \\
\text { II }\end{array}$ & Multinivel & $(+)$ & N.I & NSE: + & N.I \\
\hline Correa (2004) & $\begin{array}{l}\text { Puntuación total: } \\
\text { Saber } 11 \text { 2001-I. } \\
\text { Cali }\end{array}$ & Multinivel & $(+)$ & $(-)$ & $\begin{array}{c}\text { Ingresos } \\
\text { familiares: + }\end{array}$ & $\begin{array}{l}\text { Padre con el mayor } \\
\text { nivel educativo }(+)\end{array}$ \\
\hline $\begin{array}{l}\text { Barrientos \& } \\
\text { Ríos (2007) }\end{array}$ & $\begin{array}{l}\text { Saber } 11-1999- \\
\text { 2004-2006 }\end{array}$ & Estudiante & $(+)$ & $(-)$ & Estrato: $(+/-)$ & N.I \\
\hline Bonilla (2011) & $\begin{array}{l}\text { Puntuación total: } \\
\text { Saber } 11 \text { - } 2009\end{array}$ & Estudiante & $(+)$ & $(-)$ & $\begin{array}{c}\text { Ingresos } \\
\text { familiares: }+\end{array}$ & Madre (+) \\
\hline \multirow{2}{*}{ Barón (2012) } & $\begin{array}{l}\text { Saber } 11 \text { 2009: } \\
\text { Barranquilla }\end{array}$ & Estudiante & NA & N.I & $\begin{array}{c}\text { Ingresos } \\
\text { familiares: + }\end{array}$ & $\begin{array}{c}\text { Ambos padres }(+) \text { para } \\
\text { mujeres y mixto para } \\
\text { hombres }\end{array}$ \\
\hline & $\begin{array}{c}\text { Saber } 11 \text { 2009: } \\
\text { Bogotá }\end{array}$ & Estudiante & NA & N.I & $\begin{array}{c}\text { Ingresos } \\
\text { familiares: + }\end{array}$ & $\begin{array}{c}\text { Ambos padres }(+) \text { para } \\
\text { mujeres y hombres }\end{array}$ \\
\hline $\begin{array}{l}\text { Montes, Garcés, } \\
\text { Chica \& } \\
\text { Jaramillo (2014) }\end{array}$ & Saber $11-2010$ & Multinivel & $(+)$ & $(-)$ & NSE: + & N.I \\
\hline
\end{tabular}

Nota: N.I: Variable no se incluyó y NA: no aplica. Sólo se incluyeron los años de la base de datos de Saber 11 utilizada y la variable dependiente en caso de que no sea la puntuación en matemáticas y aquellas variables que fueron significativas al menos al $95 \%$ de confianza.

Fuente: Elaboración propia. 
En cuanto a las condiciones socioeconómicas del hogar se encuentra que cuanto mejores sean éstas, independientemente de cómo se midan, mayor será el efecto positivo sobre la puntuación en matemáticas.

Otra variable que se incluye bajo este grupo de factores es el nivel educativo de los padres (de la madre, el padre o ambos). En términos generales aquellos estudiantes cuyos padres han alcanzado un mayor nivel educativo obtienen en promedio mejores puntuaciones.

Con respecto al colegio, las variables más utilizadas son la naturaleza jurídica de la institución (si es oficial o no) y la jornada escolar como se puede observar en la Tabla 2. La naturaleza jurídica de la institución se refiere a si el colegio es financiado con recursos públicos (colegios oficiales también conocidos como colegios públicos) o con recursos privados (colegios no oficiales). Estos dos tipos de colegios se tienden a diferenciar en términos de infraestructura (mejor colegios privados), proporción de jornada completa vs jornada de la mañana o de la tarde (mayor proporción de jornada completa para colegios privados), de elementos administrativos, o del contexto de los estudiantes (Duarte, Bos \& Moreno, 2012; Gómez, 2016). Para un mejor entendimiento de la naturaleza de los colegios se sugiere revisar el libro "Revisión de políticas nacionales de educación: La educación en Colombia", elaborado por la OCDE en conjunto con el Ministerio de Educación Nacional se explican las características de los colegios oficiales en Colombia y cómo se diferencian de los colegios privados, así como el tipo de establecimiento educativo entre las categorías de municipios.

Además, otra diferencia resulta en que los padres no pueden seleccionar directamente el colegio público al que sus hijos van a asistir. Cuando hacen la inscripción por primera vez o necesitan hacer un cambio porque se mudaron, deben completar una solicitud ante el Secretario de Educación del municipio. En esta aplicación, los padres pueden expresar su preferencia por algunas escuelas, pero eso no significa que el colegio al que asigne al estudiante corresponda a las preferencias de los padres, porque decisión depende de la demanda y la oferta espacios escolares para cada grado (Hincapié, 2016).

Tabla 2. Factores del colegio asociados a la puntuación en matemáticas.

\begin{tabular}{|c|c|c|c|c|}
\hline \multirow{2}{*}{ Autor } & \multirow{2}{*}{ Datos } & \multirow{2}{*}{ Modelo } & \multicolumn{2}{|c|}{ Factores del colegio } \\
\hline & & & Oficial & Jornada escolar \\
\hline \multirow{3}{*}{$\begin{array}{c}\text { Piñeros \& Rodríguez } \\
\text { (1998) }\end{array}$} & \multirow{3}{*}{ Saber 11 - 1997 II } & \multirow{3}{*}{ Multinivel } & \multirow{3}{*}{ N.I. } & Mañana (+) \\
\hline & & & & Tarde $(+)$ \\
\hline & & & & Completa $(+)$ \\
\hline \multirow{3}{*}{ Correa (2004) } & \multirow{3}{*}{$\begin{array}{l}\text { Puntuación total: } \\
\text { Saber } 11 \text { 2001-I. } \\
\text { Cali }\end{array}$} & \multirow{3}{*}{ Multinivel } & \multirow{3}{*}{$(+)$} & Mañana (+) \\
\hline & & & & Tarde (-) \\
\hline & & & & Noche (-) \\
\hline Barrientos \& Ríos (2007) & $\begin{array}{l}\text { Saber } 11 \text { 1999- } \\
2004-2006\end{array}$ & Estudiante/Cuartil & N.I. & $\begin{array}{c}\text { Tarde }(+): 0,25,0,5 \mathrm{y} \\
0,75\end{array}$ \\
\hline Bonilla (2011) & $\begin{array}{l}\text { Puntuación total / } \\
\text { Saber } 11-2009\end{array}$ & Estudiante & $(+)$ & Completa $(+)$ \\
\hline Barón (2012) & $\begin{array}{l}\text { Saber } 11 \text { 2009: } \\
\text { Barranquilla y } \\
\text { Bogotá }\end{array}$ & Estudiante & N.I. & Completa o mañana $(+)$ \\
\hline \multirow{3}{*}{$\begin{array}{l}\text { Montes, Garcés, Chica \& } \\
\text { Jaramillo (2014) }\end{array}$} & \multirow{3}{*}{ Saber 112010} & \multirow{3}{*}{ Multinivel } & \multirow{3}{*}{$(-)$} & Mañana (+) \\
\hline & & & & Tarde $(+)$ \\
\hline & & & & Completa $(+)$ \\
\hline Hincapié (2016) & $\begin{array}{c}\text { Saber } 5 \text { y } 9-2002 \\
2005, \text { y } 2009 .\end{array}$ & Estudiante & NA & Completa $(+)$ \\
\hline
\end{tabular}


Nota: N.I: Variable no se incluyó y NA: no aplica * Sólo se incluyeron los años de la base de datos de Saber 11 utilizada y la variable dependiente en caso de que no sea la puntuación en matemáticas $\uparrow$ Sólo se incluyeron aquellas variables que fueron significativas al menos al $95 \%$ de confianza.

Fuente: Elaboración propia.

Con respecto al lugar de residencia Restrepo y Alviar (2009) buscan determinar cuál es el efecto colegio sobre la variabilidad de los resultados de Saber 11 dependiendo de la subregión del departamento de Antioquia que se analice. Bonilla (2011) incluye características de diferente índole (sociales, educativas, económicas, entre otras) de las 10 ciudades principales del país y encuentra que éstas son significativas (Tabla 3).

Tabla 3. Factores del lugar de residencia asociados a la puntuación en matemáticas.

\begin{tabular}{|c|c|c|c|c|}
\hline Autor & Datos & Modelo & Municipio & Hallazgos \\
\hline $\begin{array}{l}\text { Restrepo \& Alviar } \\
\text { (2009) }\end{array}$ & Saber 11- 1999 & Multinivel & $\begin{array}{c}\text { Subregiones } \\
\text { Antioquia }\end{array}$ & $\begin{array}{c}\text { Oficial (-) } \\
\text { Jornada mañana (-) }\end{array}$ \\
\hline Bonilla (2011) & $\begin{array}{l}\text { Puntuación total } \\
\text { Saber } 11 \text { - } 2009\end{array}$ & Estudiante & $\begin{array}{l}10 \text { ciudades } \\
\text { principales }\end{array}$ & $\begin{array}{c}\% \text { Urbano (-) } \\
\text { \% NBI (-) } \\
\% \text { analfabetismo (-) } \\
\% \text { homicidios (-) } \\
\text { Desempeño fiscal (+) }\end{array}$ \\
\hline $\begin{array}{c}\text { Martínez \& } \\
\text { Turriago (2015) }\end{array}$ & $\begin{array}{c}\text { Puntuación total: } \\
\text { Saber } 11 \text { - 2005-2012, } \\
\text { semestre II }\end{array}$ & NA & Todos & $\begin{array}{c}\text { NBI }(-) \\
\text { \% Alumnos del sector privado }(+) \\
\text { \% Profesores con educación } \\
\text { superior }(+)\end{array}$ \\
\hline
\end{tabular}

Nota: N.I: Variable no se incluyó y NA: no aplica * Sólo se incluyeron los años de la base de datos de Saber 11 utilizada y la variable dependiente en caso de que no sea la puntuación en matemáticas $†$ Sólo se incluyeron aquellas variables que fueron significativas al menos al $95 \%$ de confianza.

Fuente: Elaboración propia.

Lo mismo sucede con Martínez y Turriago (2015), quienes realizan un análisis gráfico y estadístico (empleando el coeficiente de correlación y el coeficiente de determinación) y evidencian que menores índices de necesidades básicas insatisfechas y un mayor nivel de preparación de los docentes se asocian directamente con la puntuación total de los estudiantes en las pruebas.

\section{Aproximación empírica.}

Las aproximaciones empíricas utilizadas para analizar el efecto que tienen diferentes factores en el desempeño escolar son: regresiones cuantílicas (QR por sus siglas en inglés) y regresiones de modelos lineales jerárquicos. Sin embargo, la regresión cuantílica no permite analizar datos cuya naturaleza es jerárquica, como en el caso educativo, donde los estudiantes se agrupan en salones y estos a su vez en colegios. Tzavidis y Brown (2010), Geraci y Bottai (2007) y Smith et al. (2015) combinan las propiedades de las regresiones cuantílicas y los modelos lineales jerárquicos para solventar esta limitación de la regresión cuantílica. En este artículo, utilizaremos el método de regresión por cuartiles para modelos lineales jerárquicos (LQMM) de Geraci y Bottai (2014) que permite estimar el cuantil condicional de la variable dependiente en función de efectos fijos y aleatorios. Lo anterior implica: 
1. Estimar el modelo vacío o de efectos fijos (Ecuación 1) y calcular el coeficiente de correlación intraclase (Ecuación 2).

$$
\begin{gathered}
y_{i j}=1 * \theta^{\tau}+1 * u_{j}+\varepsilon_{i j}^{\tau} \\
I C C=p^{\tau}=\frac{\Psi_{u_{\tau}}^{2}}{\Psi_{u_{\tau}}^{2}+\Psi_{\varepsilon_{\tau}}^{2}}
\end{gathered}
$$

donde $\Psi_{\varepsilon_{\tau}}^{2}$ representa la varianza al interior del colegio (entre los alumnos que se encuentran en el cuantil $\tau$ ), y $\Psi_{u_{\tau}}^{2}$ representa la varianza entre colegios en el cuantil $\tau$. Luego $\Psi_{u_{\tau}}^{2}+\Psi_{\varepsilon_{\tau}}^{2}$ indica la varianza total.

2. Estimar el modelo incluyendo la variable independiente del entorno de desarrollo:

$$
y_{i j}=x_{i j}^{T} \theta^{\tau}+z_{i j}{ }^{T} u_{j}+\varepsilon_{i j}^{\tau}
$$

donde:

- $\tau$ : son los percentiles $0,25,0,5$ y 0,75

- $\mathrm{y}_{\mathrm{ijj}}$ : es la puntuación en matemáticas del estudiante i que asiste al colegio $\mathrm{j}$

- $x_{i j}^{T}=\left(1\right.$, munintermedia $_{\mathrm{ij}}$, munincipiente $\mathrm{i}_{\mathrm{ij}}$, edad $_{\mathrm{ij}}$, mujer $_{\mathrm{ij}}$, msecundaria $_{\mathrm{ij}}$, mterciaria $_{\mathrm{ij}}$, oficial $_{\mathrm{ij}}$, jmanana $\mathrm{ij}_{\mathrm{ij}}$, jtarde $\mathrm{ij}$ )

- $\theta^{\tau}=\left(\theta_{0}^{\tau}, \theta_{1}^{\tau}, \theta_{2}^{\tau}, \theta_{3}^{\tau}, \theta_{4}^{\tau}, \theta_{5}^{\tau}, \theta_{6}^{\tau}, \theta_{7}^{\tau}, \theta_{8}^{\tau}, \theta_{9}^{\tau}\right)^{T}$

- $z_{\mathrm{ij}}: 1$

- $\quad \mathrm{u}_{\mathrm{j}}$ : son los efectos aleatorios en el intercepto $\mathrm{u} \perp \varepsilon_{\mathrm{ij}} \mathrm{y} \mathrm{u} \sim \mathrm{N}\left(0, \Psi^{\tau}\right)$ por lo tanto las estimaciones se calcularán mediante integración numérica utilizando el método de cuadratura Gauss- Hermite (Geraci \& Bottai, 2014).

- $\varepsilon$ : término de error $\varepsilon \sim \operatorname{Al}(0, \sigma, \tau)$.

3. Estimar el modelo incluyendo la variable independiente de las tipologías municipales que conforman los entornos de desarrollo:

$$
y_{i j}=x_{i j}^{T} \delta^{\tau}+z_{i j}{ }^{T} u_{j}+\varepsilon_{i j}{ }^{\tau}
$$

donde:

- $x_{i j}^{T}=\left(1, \mathrm{~B}_{\mathrm{ij}}, \mathrm{C}_{\mathrm{ij}}, \mathrm{D}_{\mathrm{ij}}, \mathrm{E}_{\mathrm{ij}}, \mathrm{F}_{\mathrm{ij}}, \mathrm{G}_{\mathrm{ij}}\right.$, mujer $_{\mathrm{ij}}$, msecundaria $_{\mathrm{ij}}$, mterciaria $_{\mathrm{ij}}$, edad $_{\mathrm{ij}}$, oficial $_{\mathrm{ij}}$, jmanana $\left._{\mathrm{ij}}, \mathrm{jtarde}_{\mathrm{ij}}\right)$

- $\delta^{\tau}=\left(\delta_{0}^{\tau}, \delta_{1}^{\tau}, \delta_{2}^{\tau}, \delta_{3}^{\tau}, \delta_{4}^{\tau}, \delta_{5}^{\tau}, \delta_{6}^{\tau}, \delta_{6}^{\tau}, \delta_{7}^{\tau}, \delta_{8}^{\tau}, \delta_{9}^{\tau}, \delta_{10}^{\tau}, \delta_{11}^{\tau}, \delta_{12}^{\tau}, \delta_{13}^{\tau}\right)^{T}$

4. Estimar el modelo de la ecuación 4 empleando una regresión cuantílica y un modelo lineal jerárquico.

Los errores estándar para determinar la significancia individual de los coeficientes estimados se obtienen por el método de bootstraping (se emplean 500 repeticiones). 


\section{Los datos.}

Por un lado, las tipologías y clasificaciones del entorno de desarrollo municipal provienen de la propuesta elaborada por el DNP (Bogotá D.C. no se incluyó en la Tipología Municipal sino en la Tipología Departamental, por lo que de ésta se extrajo la información correspondiente). Esta tipología se desarrolló para facilitar el diseño de políticas públicas, de tal forma que se pueda responder de manera asertiva e integral a las necesidades de las diferentes entidades territoriales. La metodología empleada para construir las tipologías se basó en el análisis de componentes principales (Aguilar et al., 2015). De lo anterior resultaron tres entornos de desarrollo: i) Entorno de Desarrollo Robusto que corresponde a los municipios pertenecientes a la tipología municipal A y B; ii) Entorno de Desarrollo Intermedio corresponde a los municipios que pertenecen a la tipología C, D y E; iii) Entorno Incipiente, son los municipios pertenecientes a la tipología F y G.

Como se observa en la Tabla 4, si bien la tipología A agrupa la menor cantidad de municipios del país $(0,5 \%)$, contiene casi un tercio de los habitantes del país para el 2014. Las otras tipologías contienen un menor número de habitantes, siendo la tipología $\mathrm{G}$ la que contiene la menor cantidad (3.6\% del total de habitantes). Por otro lado, entre todas las tipologías, es la E la que agrupa la mayor cantidad de municipios, seguida de la D, F y C.

Tabla 4. Número de municipios y habitantes por Tipología municipal.

\begin{tabular}{ccccccc}
\hline $\begin{array}{c}\text { Entorno de } \\
\text { desarrollo }\end{array}$ & Tipología & $\begin{array}{c}\text { Número de } \\
\text { municipios }\end{array}$ & $\begin{array}{c}\text { Participación } \\
\text { (\%) }\end{array}$ & Población & \% Población & Ejemplo \\
\hline \multirow{2}{*}{ Robusto } & $\mathrm{A}$ & 6 & $0,5 \%$ & 15.293 .275 & $32,17 \%$ & Cali \\
& $\mathrm{B}$ & 63 & $5,7 \%$ & 9.179 .477 & $19,31 \%$ & Manizales \\
\multirow{2}{*}{ Intermedio } & $\mathrm{C}$ & 158 & $14,4 \%$ & 7.250 .124 & $15,25 \%$ & Tunja \\
& $\mathrm{D}$ & 261 & $23,7 \%$ & 5.812 .304 & $12,23 \%$ & Florencia \\
& $\mathrm{E}$ & 293 & $26,6 \%$ & 5.126 .952 & $10,79 \%$ & El Cocuy \\
\multirow{2}{*}{ Incipiente } & $\mathrm{F}$ & 209 & $19,0 \%$ & 3.173 .444 & $6,68 \%$ & Moñitos \\
& $\mathrm{G}$ & 111 & $10,1 \%$ & 1.700 .315 & $3,58 \%$ & Currillo \\
\hline
\end{tabular}

Fuente: Adaptado de Carmona, Supelano \& Osejo (2015).

Las tipologías dan información sobre las características propias de cada municipio, con relación a seis dimensiones: la urbano regional, las condiciones sociales, las condiciones económicas, las condiciones ambientales, las condiciones institucionales y de seguridad.

Con respecto a los datos de las puntuaciones en matemáticas y, las características de los estudiantes, sus familias y los colegios a los que asisten, provienen de la prueba Saber 11 2014-2, que es aplicada por el Instituto Colombiano para la Evaluación de la Educación (ICFES) a todos los estudiantes que se deseen graduar del bachillerato. De acuerdo con el ICFES (2014) la prueba Saber 11 evalúa 5 competencias: matemáticas, lectura crítica, sociales y competencias ciudadanas, ciencias naturales e inglés. Las puntuaciones de estos componentes se miden en una escala de 0 a 100 , con media 50 y desviación estándar 10.

Se seleccionó el segundo semestre de 2014 por dos razones. En primer lugar, el indicador de tipología municipal está construido sólo para el 2014. En segundo lugar, en ese semestre se introdujeron cambios a la forma y estructura de la prueba por lo que los resultados del primer semestre no son 
estrictamente comparables con los del segundo semestre (Ministerio de Educación Nacional, 2014). La base de datos de 2014 se caracteriza en términos generales por incluir:

- Estudiantes que tienen entre 14 y 28 años.

- Estudiantes que asisten a colegios públicos y privados, en jornada diurna (mañana, tarde, y completa)

- Viven en cualquier municipio de Colombia excepto en San Yrés Islas, Belén de Bajira, El Encanto, La Chorrera, La Pedrera, La Victoria, Mirití - Parana, Puerto Santyer, Tarapaca, Barranco Minas, Maripipana. Lo anterior es debido a que para estos municipios no existe una tipología definida por el DNP.

- Pertenecen a alguna institución educativa.

- Toman la prueba por primera vez.

- Libres, es decir, no están en centros penitenciarios.

- Reportaron información para todas las variables de interés.

- Pertenecen a instituciones educativas que presentaron como mínimo 10 estudiantes.

De acuerdo con lo anterior, se obtuvieron 410.608 observaciones de 533.133 ; es decir el 77,0\% de las observaciones originales. La Tabla 5 muestra las estadísticas descriptivas de todas las variables usadas en la estimación a nivel nacional y por entorno de desarrollo.

Tabla 5. Estadísticas descriptivas a nivel nacional y por entorno de desarrollo.

\begin{tabular}{lcccc}
\hline \multirow{2}{*}{\multicolumn{1}{c}{ Variable }} & \multicolumn{4}{c}{ Entorno de desarrollo } \\
\cline { 2 - 5 } & Nacional & Incipiente & Intermedio & Robusto \\
\hline No . de estudiantes & 410.709 & 35.111 & 150.928 & 224.670 \\
Personales & & & & \\
Promedio puntuación Matemáticas & 50,88 & 46,58 & 49,32 & 52,60 \\
Desviación Estándar puntuación Matemáticas & 9,68 & 8,29 & 9,04 & 9,90 \\
Primer cuartil puntuación Matemáticas & 45 & 41 & 44 & 46 \\
Segundo cuartil puntuación Matemáticas & 50 & 46 & 49 & 52 \\
Tercer cuartil puntuación Matemáticas & 56 & 52 & 55 & 58 \\
Mujer (\%) & 54,89 & 53,30 & 55,61 & 54,65 \\
Edad (promedio años) & 16,82 & 17,14 & 16,87 & 16,74 \\
Familiares (\%) & & & & \\
Nivel educativo madre: ninguno o primaria & 33,21 & 58,14 & 43,11 & 22,67 \\
Nivel educativo madre: secundaria & 45,18 & 33,34 & 41,45 & 49,53 \\
Nivel educativo madre: terciaria & 21,61 & 8,52 & 15,44 & 27,80 \\
Colegio (\%) & & & & \\
Oficial & 77,47 & 97,82 & 90,51 & 65,54 \\
Jornada Mañana & 59,63 & 77,23 & 64,86 & 53,37 \\
Jornada Tarde & 18,36 & 8,40 & 14,45 & 22,55 \\
Jornada Completa & 21,98 & 14,36 & 20,64 & 24,07 \\
\hline
\end{tabular}

Fuente: Elaboración propia. 
En cuanto a las características personales, se puede observar que la puntuación promedio en matemáticas de los estudiantes que viven en los entornos de desarrollo incipiente e intermedio está por debajo de la puntuación promedio nacional; mientras que, el promedio en el entorno robusto está por encima. Este comportamiento se mantiene si se comparan las puntuaciones en matemáticas obtenidos por cuartil, tanto por entornos de desarrollo como en el ámbito nacional.

De igual forma hay diferencias al interior de los entornos. Por ejemplo, se observa que la diferencia entre la cola inferior (primer cuartil) y la cola superior (tercer cuartil) de la distribución de la puntuación aumenta ligeramente por entorno de desarrollo: 9 puntos para entorno de desarrollo incipiente; 11 puntos para entorno de desarrollo intermedio; y 12 para entorno de desarrollo robusto.

Por otra parte, el porcentaje de mujeres es relativamente similar y superior al $50 \%$ para todos los casos. La edad promedio del estudiante es de 16,82 años a nivel nacional, para el municipio incipiente e intermedio es ligeramente mayor y para los estudiantes de municipios robustos es menor.

En cuanto a las características familiares, en el ámbito nacional el $45,18 \%$ de los estudiantes reportaron que el máximo nivel educativo alcanzado por su madre había sido secundaria (incompleta o completa); en este mismo nivel educativo se concentra la mayoría de respuestas de los estudiantes que viven en un entorno de desarrollo robusto. Por el contrario, tanto en el entorno incipiente como en el intermedio el mayor porcentaje de las respuestas se concentró en ninguno o primaria (incompleta o completa).

Finalmente, más del 90\% de los estudiantes que viven en un entorno de municipio incipiente o intermedio asisten a colegios oficiales, mientras que en los municipios robustos ese porcentaje es del $65,5 \%$. En los tres entornos, más del 50\% de los estudiantes asiste a la jornada de la mañana, seguido de jornada completa $y$, por último, la jornada de la tarde.

\section{Resultados.}

En esta sección se presentan los resultados de la aplicación de la aproximación empírica a nivel de entorno de desarrollo. Los resultados iniciales indican que el colegio explica el $64 \%$ de la variación en los resultados de la puntuación en matemáticas de los estudiantes que se encuentran en la cola inferior de la distribución. Este porcentaje aumenta en 12 puntos porcentuales si el estudiante se encuentra en la mediana de la distribución y en 4 puntos porcentuales para el estudiante del tercer cuartil con respecto al primer cuartil. Lo anterior apoya la hipótesis de que es adecuado emplear modelos lineales jerárquicos.

Tabla 6. Coeficiente de correlación interclase del modelo vacío.

\begin{tabular}{cc}
\hline Cuartil & CCI (\%) \\
\hline 0,25 & 64 \\
0,50 & 76 \\
0,75 & 68 \\
\hline \multicolumn{2}{c}{ Fuente: Elaboración propia. }
\end{tabular}

La Tabla 7 muestra los resultados de la estimación del modelo jerárquico lineal por cuartiles (LQMM), del modelo lineal jerárquico (HML) y de la regresión cuantílica (RQ). El hecho de que los factores del colegio expliquen un alto porcentaje de las variaciones en las puntuaciones en matemáticas indica que hay algunos colegios que alcanzan unos altos niveles de desempeño, mientras que otros alcanzan desempeños muy bajos. Lo que podría estar indicando que en términos de equidad en el área educativa todavía hay un largo camino que recorrer (Foy, 2004). Estos resultados son acordes con los 
estudios que revelan que la asignación de recursos físicos y humanos en el área educativa no están distribuidos equitativamente entre las entidades territoriales (Bonet, 2005; Bonilla, 2011).

Tabla 7. Estimación modelo jerárquico lineal, modelo jerárquico lineal por cuantiles y regresión cuantílica por entornos de desarrollo.

\begin{tabular}{|c|c|c|c|c|c|c|c|}
\hline \multirow{2}{*}{ Variable/Método } & \multirow{2}{*}{ HML } & \multicolumn{3}{|c|}{ LQMM } & \multicolumn{3}{|c|}{ RQ } \\
\hline & & Cuartil 1 & Cuartil 2 & Cuartil 3 & Cuartil 1 & Cuartil 2 & Cuartil 3 \\
\hline \multicolumn{8}{|l|}{ Efectos fijos } \\
\hline \multirow{2}{*}{ Intercepto } & $55,27 * * *$ & $49,91 * * *$ & $55,38 * * *$ & $60,37 * * *$ & $49,68 * * *$ & $54,72 * * *$ & $60,41 * * *$ \\
\hline & 0,11 & 0,66 & 0,53 & 0,34 & 0,43 & 0,07 & 0,19 \\
\hline \multirow{2}{*}{$\begin{array}{l}\text { Entorno de } \\
\text { desarrollo } \\
\text { Intermedio }\end{array}$} & $-2,08 * * *$ & $-1,35 * * *$ & $-2,00 * * *$ & $-2,34 * * *$ & $-1,89 * * *$ & $-1,96 * * *$ & $-1,98 * * *$ \\
\hline & 0,09 & 0,38 & 0,25 & 0,27 & 0,13 & 0,05 & 0,07 \\
\hline \multirow{2}{*}{$\begin{array}{l}\text { Entorno de } \\
\text { desarrollo Incipiente }\end{array}$} & $-3,39 * * *$ & $-3,0 * * *$ & $-3,25 * * *$ & $-4,02 * * *$ & $-3,47 * * *$ & $-3,75 * * *$ & $-3,86^{* * *}$ \\
\hline & 0,14 & 0,52 & 0,41 & 0,44 & 0,12 & 0,06 & 0,12 \\
\hline \multicolumn{8}{|c|}{ Efectos fijos: controles } \\
\hline \multirow{2}{*}{ Mujer } & $-2,83 * * *$ & $-2,00 * * *$ & $-2,74 * * *$ & $-3,18 * * *$ & $-2,00 * * *$ & $-2,49 * * *$ & $-3,00 * * *$ \\
\hline & 0,03 & 0,08 & 0,16 & 0,08 & 0,09 & 0,02 & 0,01 \\
\hline \multirow{2}{*}{ Edad (centrada) } & $-1,59 * * *$ & $-1,35 * * *$ & $-1,50 * * *$ & $-1,67 * * *$ & $-1,50 * * *$ & $-1,52 * * *$ & $-1,86^{* * *}$ \\
\hline & 0,02 & 0,18 & 0,03 & 0,06 & 0,02 & 0,02 & 0,05 \\
\hline \multirow{2}{*}{$\begin{array}{l}\text { Nivel educativo } \\
\text { madre: secundaria }\end{array}$} & $0,80 * * *$ & $0,65 * * *$ & $0,75 * * *$ & $0,83 * * *$ & $0,50 * * *$ & $1,02 * * *$ & $1,41 * * *$ \\
\hline & 0,03 & 0,16 & 0,16 & 0,11 & 0,10 & 0,04 & 0,17 \\
\hline \multirow{2}{*}{$\begin{array}{l}\text { Nivel educativo } \\
\text { madre: terciaria }\end{array}$} & $2,87 * * *$ & $2,65 * * *$ & $2,74 * * *$ & $3,15 * * *$ & $3,50 * * *$ & $4,88 * * *$ & $6,37 * * *$ \\
\hline & 0,04 & 0,23 & 0,16 & 0,15 & 0,15 & 0,06 & 0,16 \\
\hline \multirow{2}{*}{ Oficial } & $-1,46^{* * *}$ & $-1,97 * * *$ & $-1,55 * * *$ & $-1,78 * * *$ & $-3,43 * * *$ & $-3,47 * * *$ & $-3,68 * * *$ \\
\hline & 0,07 & 0,31 & 0,23 & 0,14 & 0,22 & 0,09 & 0,14 \\
\hline \multirow{2}{*}{ Jornada mañana } & $-2,09 * * *$ & $-2,03 * * *$ & $-2,45 * * *$ & $-1,87 * * *$ & $-0,13$ & $-0,16^{*}$ & $-0,22 * *$ \\
\hline & 0,12 & 0,70 & 0,55 & 0,33 & 0,09 & 0,07 & 0,09 \\
\hline \multirow{2}{*}{ Jornada tarde } & $-2,66^{* * *}$ & $-2,38 * * *$ & $-2,71 * * *$ & $-2,05 * * *$ & $-0,63 * * *$ & $-0,68 * * *$ & $-0,95 * * *$ \\
\hline & 0,15 & 1,06 & 0,76 & 0,48 & 0,13 & 0,07 & 0,09 \\
\hline \multicolumn{8}{|l|}{ Efectos aleatorios } \\
\hline Colegio (sd) & 3,95 & 3,19 & 2,95 & 3,26 & & & \\
\hline $\mathrm{CCI}$ & $20,00 \%$ & $53,32 \%$ & $58,63 \%$ & $58,96 \%$ & & & \\
\hline
\end{tabular}

El intercepto, es significativo y positivo para todos los cuartiles, y se puede interpretar como la puntuación en matemáticas promedio respectivo a cada cuartil que obtendría un estudiante hombre con 16,82 años en promedio, cuya madre no ha alcanzado un nivel educativo o sólo alcanzó la primaria (completa o incompleta), que vive en un entorno de desarrollo robusto, asiste a un colegio no oficial en la jornada de completa. De esta forma, un estudiante con estas características obtendría una puntuación de 55,27 puntos en los resultados de matemáticas siguiendo el modelo HML y un de entre 49,912 puntos y 60,37 puntos usando el modelo LQMM. Los resultados en los modelos LQMM y RQ muestran que la magnitud del coeficiente aumenta a medida que se pasa de cuartil de estudio, que es un resultado esperado ya que el último cuartil es el de aquellos estudiantes con mejores resultados en la prueba Saber 
11. El intercepto del modelo HML también es significativo, pero por su misma naturaleza oculta las diferencias en el desempeño que existen a lo largo de la distribución. Se realizaron regresiones excluyendo los outliers y los resultados no son sustancialmente diferentes a los aquí reportados.

Los resultados muestran que vivir en un municipio catalogado como de un entorno de desarrollo incipiente o intermedio tiene un efecto negativo en el desempeño en matemáticas comparado con vivir en un municipio de entorno de desarrollo robusto. Lo anterior también se evidencia con la aplicación del modelo HML y RQ. Por otra parte, un estudiante el primer cuartil $(0,25)$ que viva en un entorno de desarrollo incipiente obtiene en promedio tres puntos menos que aquel que vive en un entorno de desarrollo robusto, esa diferencia es mayor para aquellos que se encuentran en el segundo o tercer cuartil.

Aquellos individuos con más edad tienen un desempeño en matemáticas menor a sus pares más jóvenes. Las mujeres tienen un desempeño menor al de los hombres. De hecho, se puede observar que el ser mujer afecta más a los estudiantes de alto desempeño (tercer cuartil). El nivel educativo alcanzado por la madre también es positivo y estadísticamente significativo. Un estudiante cuya madre haya alcanzado un nivel terciario de educación obtiene en promedio 2,7 puntos adicionales si se compara con un estudiante cuya madre no alcanzó algún nivel educativo o sólo alcanzó la primaria.

Con respecto a las variables del colegio, se encuentra que asistir a un colegio oficial está correlacionado negativa y significativamente sólo con aquellos estudiantes que se encuentran en la cola superior. Resultados de otros estudios han encontrado, para el caso colombiano, que esto puede obedecer no solamente a unas mejores condiciones socioeconómicas de los estudiantes que asisten a estos colegios, sino también a la forma en que son administrados y a la estructura de incentivos que se maneja al interior de estos (Gaviria \& Barrientos, 2001).

Los resultados también muestran que los estudiantes que asisten a la jornada de mañana o de la tarde tienen un menor desempeño que aquellos que asisten a la jornada completa. Ese efecto negativo es mayor para aquellos que asisten a la jornada de la tarde que para aquellos que asisten a la jornada de la mañana.

Finalmente, se puede observar que los coeficientes de correlación interclase para el modelo LQMM disminuyeron con respecto a los obtenidos con el modelo vacío (60\%). En el caso del primer cuartil disminuyó 7 puntos, lo que es explicado por las covariables incluidas en el modelo.

Ahora bien ¿existen diferencias estadísticamente significativas entre los cuartiles de la distribución? O, mejor dicho ¿el efecto de las covariables es estadísticamente diferente a través de la distribución de las puntuaciones? Esto sin duda nos ayuda a comparar si, por ejemplo, el colegio tiene algún efecto estadísticamente significativo a lo largo de la distribución de las puntuaciones. Es decir, entre los estudiantes con mejor desempeño (75\%) versus, por ejemplo, aquellos que tienen un resultado inferior (25\%). Agradecemos los comentarios de uno de los evaluadores al respecto.

Con el fin de determinar si existe diferencia estadísticamente significativa entre los cuartiles de los coeficientes estimados para cada variable se utilizó el contraste propuesto por Clogg, Petkova y Haritou (1995) y Paternoster et al. (1998):

$$
Z=\frac{\beta_{i}-\beta_{j}}{\sqrt{S d_{i}^{2}+S d_{j}^{2}}}
$$

donde $\beta_{i} y \beta_{j}$ son los coeficientes estimados en el cuartil i y el cuartil j y $S d_{i}^{2} y S d_{j}^{2}$ son los errores estándar del coeficiente en el cuartil i y los errores estándar del coeficiente en el cuartil. Los resultados aparecen en la Tabla 8. 
Tabla 8. Contraste sobre diferencias entre cuartiles.

\begin{tabular}{|c|c|c|c|c|c|c|}
\hline \multirow{2}{*}{ Variable/Modelo } & \multicolumn{2}{|c|}{ Test $(q 1 / q 3)$} & \multicolumn{2}{|c|}{ Test (q1/q2) } & \multicolumn{2}{|c|}{ Test (q2/q3) } \\
\hline & RQ & LQMM & RQ & LQMM & RQ & LQMM \\
\hline \multicolumn{7}{|l|}{ Efectos fijos } \\
\hline Intercepto & $-22,82 * * *$ & $-14,09 * * *$ & $-11,57 * * *$ & $-6,46 * *$ & $-28,10 * * *$ & $-7,92 * * *$ \\
\hline Entorno de desarrollo & & & & & & \\
\hline Intermedio & 0,61 & $2,13 * * *$ & 0,50 & 1,43 & 0,23 & 0,92 \\
\hline Entorno de desarrollo & & & & & & \\
\hline Incipiente & $2,29 * *$ & 1,50 & $2,09 * *$ & 0,38 & 0,82 & 1,28 \\
\hline \multicolumn{7}{|l|}{ Efectos fijos: Controles } \\
\hline Mujer & $11,04 * * *$ & $10,43 * * *$ & $5,31 * * *$ & $4,17 * * *$ & $22,82 * * *$ & $2,46 * * *$ \\
\hline Edad (centrada) & $6,69 * * *$ & $1,69 * *$ & 0,71 & 0,82 & $6,31 * * *$ & $2,54 * * *$ \\
\hline $\begin{array}{l}\text { Nivel educativo madre: } \\
\text { secundaria }\end{array}$ & $-4,61 * * *$ & $-0,93$ & $-4,83 * * *$ & $-0,44$ & $-2,23 * *$ & $-0,41$ \\
\hline $\begin{array}{l}\text { Nivel educativo madre: } \\
\text { terciaria }\end{array}$ & $-13,09 * * *$ & $-1,82 * *$ & $-8,54 * * *$ & $-0,32$ & $-8,72 * * *$ & $-1,87 * *$ \\
\hline Oficial & 0,96 & $-0,56$ & 0,17 & $-1,09$ & 1,26 & 0,85 \\
\hline Jornada mañana & 0,71 & $-0,21$ & 0,26 & 0,47 & 0,53 & $-0,90$ \\
\hline Jornada tarde & $2,03 * *$ & $-0,28$ & 0,34 & 0,25 & $2,37 * *$ & $-0,73$ \\
\hline
\end{tabular}

Con respecto al entorno de desarrollo, se pude observar que existen diferencias estadísticamente significativas, dependiendo de la comparación entre cuartiles solo para el caso del entorno de desarrollo incipiente. En las regresiones por cuartiles no existe diferencia estadísticamente significativa a través de los cuartiles para la variable de desarrollo intermedio. Con respecto a las variables mujer, nivel educativo de la madre (secundaria y terciara) y jornada de la tarde se encuentran diferencias estadísticamente significativas entre los cuartiles. En el caso de la variable oficial y jornada de la mañana no se encuentra evidencia de que existan diferencias estadísticamente significativas entre los cuartiles en ambos tipos de regresión.

\section{Conclusiones.}

Los resultados muestran una relación estadística significativa entre el entorno de desarrollo y el desempeño en matemáticas. Es por esta razón, que los hacedores de políticas deben tener en cuenta las condiciones del lugar donde viven los estudiantes a la hora de formular políticas educativas encaminadas a mejorar la calidad educativa. Especialmente, deberían enfocarse en atender las necesidades de los municipios de entorno de desarrollo incipiente (tipología $\mathrm{F}$ y $\mathrm{G}$ ) y en estudiar las prácticas pedagógicas y administrativas que se imparten en los municipios del entorno de desarrollo robusto (tipología A y B). De igual forma, la política educativa debe ser diferenciada entre los estudiantes, ya que el entorno de desarrollo y las características de los colegios no afectan en la misma magnitud a todos los estudiantes.

Las diferencias, entre la puntuación en matemáticas para los estudiantes que se encuentra en el $25 \%$ peor (primer cuartil) vs el $25 \%$ mejor (estudiantes por encima del tercer cuartil) con respecto al entorno de desarrollo incipiente son estadísticamente significativas (Tabla 8). Es decir, se acentúan las diferencias para los estudiantes en municipios con un tipo de desarrollo incipiente.

De esta forma, nuestros resultados muestran que, si no se realizan acciones, el estatus quo incentiva la reproducción de una estructura social inequitativa ya que los estudiantes de bajos recursos tienen menores oportunidades para acceder a la educación superior y, por ende, tienden a repetir la baja productividad que caracteriza a sus padres; lo contrario sucede para aquellos con mejores condiciones 
socioeconómicas. El problema de tener una estructura social inequitativa que se regenera a sí misma se vuelve mayúsculo cuando se tiene en cuenta que en los 1.117 municipios restantes, donde vive el 73,3\% de la población de estudiantes de educación media según las pruebas Saber 11 2014-2, el 96,2 \% de los estudiantes son de bajos recursos. Cuantificar la magnitud de ese potencial obstáculo en el desarrollo de la industria moderna del país requiere de su estudio respectivo, pero estas cifras ponen de manifiesto la importancia de incluir en estos análisis a los municipios de los entornos de desarrollo intermedio e incipiente.

Finalmente, existen una serie de limitaciones en este artículo que podrían considerarse en próximas investigaciones. En primer lugar, hay variables a nivel de colegio y de la familia que no se tuvieron en cuenta por restricciones de los datos (los años de experiencia de los profesores, tamaño de la clase, salarios de los profesores o la mensualidad del colegio, el estrato socioeconómico o el ingreso de la familia). En segundo lugar, no se diferenciaron los colegios privados que prestan su servicio al sector educativo público, de los que no (Godoy, 2013). Y, en tercer lugar, aunque hemos incluido una conceptualización del entorno de desarrollo, desconocemos cuál o cuáles dimensiones tienen una asociación más fuerte con el desempeño y, por lo tanto, en qué condición o característica del lugar donde reside el estudiante se deberían focalizar la inversión pública. De igual forma, no se incluyeron componentes que determinan la calidad educativa como cobertura, gestión pedagógica y gestión administrativa, y de los resultados solo se incluye la puntuación en matemáticas, dejando por fuera los resultados del componente de lectura crítica, sociales y ciudadanas, inglés y ciencias naturales.

\section{Referencias}

Aguilar, C.M., Osejo, I., Carmona, C., Supelano, D., Gaitán, J., \& Taborda, J. C. (2015). Tipologías municipales de Colombia: herramienta para la consolidación de regiones desarrolladas. Boletín $\begin{array}{lllll}\text { Regional, } \quad \text { Urbano } & y & \text { Ambiental, } & \text { 39-69. }\end{array}$ http://repositorio.ipea.gov.br/bitstream/11058/6172/1/BRU_n11e_tipologias.pdf

Barón, J. D. (2012). Diferencias en las características de los estudiantes y la brecha de rendimiento académico entre Barranquilla y Bogotá: Una descomposición semiparamétrica. Ensayos sobre Política Económica, 30(68), 164-215.

Barrientos, J.H., \& Ríos, P.A. (2007). Evaluación de la gestión privada del servicio público educativo en Medellín. Lecturas de Economía, (66), 147-172.

Bassett, G., Tam, M., \& Knight, K. (2002). Quantile models and estimators for data analysis. Metrika, 55(1-2), 17-26.

Bonet-Morón, J.A. (2005). Inequidad espacial en la dotación educativa regional en Colombia. Ensayos Sobre Política Económica, 51(2), 98-128.

Bonilla, L. (2011). Diferencias regionales en la distribución del ingreso en Colombia. Sociedad y Economía, 108, 43-68.

Carmona, C., Supelano, D., \& Osejo, I. (2015). Tipologías Departamentales y Municipales: Una propuesta para comprender las entidades territoriales colombianas. https://colaboracion.dnp.gov.co/CDT/Desarrollo\%20Territorial/TipFormatoPublicacion\%20\%28tipolog\%C3\%ADas\%29.pdf

Chiu, M.M., \& Xihua, Z. (2008). Family and motivation effects on mathematics achievement: Analyses of students in 41 countries. Learning and Instruction, 18(4), 321336. https://doi.org/10.1016/j.learninstruc.2007.06.003 
Clogg, C.C., Petkova, E., \& Haritou, A. (1995). Statistical methods for comparing regression coefficients between models. American Journal of Sociology, 100(5), 1261-1293.

Cooray, A.V. (2009). The role of education in economic growth. Proceedings of the 2009 Australian Conference of Economists, 1-27. Adelaide, Australia: South Australian Branch of the Economic Society of Australia.

Correa, J.J. (2004) Determinantes del rendimiento educativo de los estudiantes de secundaria en Colombia: un análisis multinivel. Revista Sociedad y Economía, 6, 81-115.

Delgado, M., Henderson, D., \& Parmeter, C. (2014). Does education matter for economic growth? Oxford Bulletin of Economics and Statistics, 76(3), 334-359.

Demir, I., Kiliç, S., \& Ünal,. H. (2010). Effects of students' and schools' characteristics on mathematics achievement: Findings from PISA 2006. Procedia - Social and Behavioral Science, 2(2), 3099-3103.

Department for Education and Skills (1999). Improving literacy and numeracy: a fresh start. The report of the working group chaired by Sir Claus Moser. Nottingham: DfES.

Duarte, J., Bos, M.S., \& Moreno, J. M. (2012). Quality, Equality and Equity in Colombian Education. Analysis of the SABER 2009 Test, (IDB-TN-396). IDB Technical Note. https://publications.iadb.org/publications/english/document/Quality-Equality-and-Equity-inColombian-Education-(Analysis-of-the-SABER-2009-Test).pdf

Eide, E., \& Showalter, M.H. (1998). The effect of school quality on student performance: A quantile regression approach. Economics letters, 58(3), 345-350. https://doi.org/10.1016/S01651765(97)00286-3

Foy, P. (2004). Intraclass correlation and variance components as population attributes and measures of sampling efficiency in PIRLS 2001. IEA Data Processing Centre, 1-13. https://www.iea.nl/sites/default/files/2019-03/IRC2004_Foy.pdf

Fryer, R.G., \& Levitt, S.D. (2010). An empirical analysis of the gender gap in mathematics. American Economic Journal: Applied Economics, 2(2), 210-240.

Galvis, L.A., \& Bonilla L. (2012). Desigualdades regionales en el nivel educativo de los profesores en Colombia. Revista de Economía Institucional, 14(26), 223-240.

Gaviria, A., \& Barrientos, J. (2001). Características del plantel y calidad de la educación en Bogotá. Coyuntura Social, 25, 81-98.

Geraci, M., \& Bottai, M. (2007). Quantile regression for longitudinal data using the asymmetric Laplace distribution. Biostatistics, 8(1), 140-154. https://doi.org/10.1093/biostatistics/kxj039

Geraci, M. \& Bottai, M. (2014). Linear quantile mixed models. Statistics and computing, 24(3), 461479. https://doi.org/10.1007/s11222-013-9381-9

Godoy, S. (2013). Análisis del programa de ampliación de cobertura en Cali con respecto a la calidad de la educación, medido a través de las pruebas de Estado. No. 011197. Departamento Nacional de Planeación.

https://repository.icesi.edu.co/biblioteca_digital/bitstream/10906/82360/1/godoy_analisis_program a_2013.pdf

Gómez, C.A. (2016). Clasificación de colegios según las pruebas Saber 11 del ICFES: un análisis usando modelos marginales (MM). Sociedad y Economía, 30, 69-89. 
Haile, G.A., \& Nguyen, A.N. (2008). Determinants of academic attainment in the United States: A quantile regression analysis of test scores. Education Economics, 16(1), 29-57. https://doi.org/10.1080/09645290701523218

Hanushek, E.A. (1986). The economics of schooling: Production and efficiency in public schools. Journal of Economic Literature, 24(3), 1141-1177.

Hanushek, E. A. (2002). Evidence, politics, and the class size debate. En L. Mishel \& R. Rothstein (Eds.), The Class Size Debate (pp. 37-66). Washington, DC: Economic Policy Institute.

Hanushek, E.A. (2013). Economic growth in developing countries: The role of human capital. Economics of Education Review, 37, 204-212. https://doi.org/10.1016/j.econedurev.2013.04.005

Hanushek, E.A., Machin, S.J., \& Woessmann, L. (Eds.). (2016). Handbook of the economics of education. Elsevier.

Hanushek, E.A., \& Woessmann, L. (2008). The role of cognitive skills in economic development. Journal of Economic Literature, 46(3), 607-668.

Hincapie, D. (2016). Do longer school days improve student achievement? Evidence from Colombia (No. IDB-WP-679). IDB Working paper series. https://publications.iadb.org/publications/english/document/Do-Longer-School-Days-ImproveStudent-Achievement-Evidence-from-Colombia.pdf

Hoerandner, C.M., \& Lemke, R.J. (2006). Can No Child Left Behind close the gaps in pass rates on standardized tests? Contemporary Economic Policy, 24(1), 1-17. https://doi.org/10.1093/cep/byj008

Instituto Colombiano para la Evaluación de la Educación [ICFES] (2014). Guía para la lectura e interpretación de los reportes de resultados dirigida a secretarias de educación. Examen de Estado Saber https://www.icfes.gov.co/documents/20143/194034/Guia\%20lectura\%20e\%20interpretacion\%20r eportes\%20resultados\%20para\%20secretarias\%20educacion\%20Saber\%2011.pdf

Jimenez, E., Lockheed, M.E., \& Paqueo, V. (1991). The relative efficiency of private and public schools in developing countries. The World Bank Research Observer, 6(2), 205-218. https://doi.org/10.1093/wbro/6.2.205

Lavy, V. (2015). Do differences in schools' instruction time explain international achievement gaps in Math, Science and Reading? Evidence from developed and developing countries. The Economic Journal, 125(588), 397-424. https://doi.org/10.1111/ecoj.12233

Lubienski, C. \& Lubienski, S. T. (2006). School Sector and Academic Achievement: A Multilevel Analysis of NAEP Mathematics Data. American Educational Research Journal, 43(4), 651-698. https://doi.org/10.3102/00028312043004651

Martínez, W.A., \& Turriago, Á. (2015). Análisis de distribución geográfica y espacial de los resultados de las Pruebas Saber 11 del Instituto Colombiano para el Fomento de la Educación Superior (ICFES). Cuadernos Latinoamericanos de Administración, 11(21), 39-50. https://doi.org/10.18270/cuaderlam.v11i21.1618

Montes, I., Garcés, J.D., Chica, S.M., \& Jaramillo, A. (2014). Rendimiento académico: ¿qué papel juegan los factores institucionales? Universidad EAFIT. https://repository.eafit.edu.co/bitstream/handle/10784/2687/2014_14_Alberto_Jaramillo.pdf;seque nce $=1$ 
Ministerio de Educación Nacional [MinEducación] (2014). El ICFES cambia la clasificación de resultados de planteles. https://www.icfes.gov.co/documents/20143/230420/Boletin\%20$\% 20$ Icfes $\% 20$ cambia\%20clasificacion\%20resultados\%20planteles.pdf

Organización para la Cooperación y el Desarrollo Económicos [OCDE] (2007). El programa PISA de la OCDE: qué es y para qué sirve. OCDE, París. https://www.oecd.org/pisa/39730818.pdf

Organización para la Cooperación y el Desarrollo Económicos [OCDE] (2014). PISA 2012: Technical Report. OCDE, París. https://www.oecd.org/pisa/pisaproducts/PISA-2012-technical-reportfinal.pdf

Paternoster, R., Brame, R., Mazerolle, P. \& Piquero, A. (1998). Using the correct statistical test for the equality of regression coefficients. Criminology, 36(4), 859-866. https://doi.org/10.1111/j.17459125.1998.tb01268.x

Piñeros, L.J. \& Rodríguez, A. (1998). Los Insumos Escolares en la Educación Secundaria y su Efecto Sobre el Rendimiento Académico de los Estudiantes: Un estudio en Colombia, LCSHD Paper Series No. 36, Human Development Department, The World Bank, Washington D.C.

Restrepo, P.P. \& Alviar, M. (2009). El logro académico y el efecto colegio en las pruebas ICFES en Antioquia. Lecturas de Economía, 60, 67-95. https://bibliotecadigital.udea.edu.co/bitstream/10495/3869/1/RestrepoPiedad_2004_LogroAcadem ico.pdf

Rivkin, S.G. \& Schiman, J.C. (2015). Instruction time, classroom quality, and academic achievement. The Economic Journal, 125(588), 425-448. https://doi.org/10.1111/ecoj.12315

Rodríguez, E. (1994). Criterios de análisis de la calidad en el sistema escolar y sus dimensiones. Revista Iberoamericana de Educación, 5, 45-65. https://doi.org/10.35362/rie501218

Smith, A. (2004). Making mathematics count. The report of Professor Adrian Smith's inquiry into post14 mathematics education. The Stationery Office Limited.

Smith, L.B., Fuentes, M., Gordon-Larsen, P., \& Reich, B.J. (2015). Quantile regression for mixed models with an application to examine blood pressure trends in China. The Annals of Applied Statistics, 9(3), 1226-1246. https://doi.org/10.1214/15-AOAS841

Tzavidis, N., \& Brown, J. J. (2010). Using M-quantile models as an alternative to random effects to model the contextual value-added of schools in London. DoQSS Working Papers 1011, Department of Quantitative Social Science, Institute of Education, University of London. https://repec.ucl.ac.uk/REPEc/pdf/qsswp1011.pdf

Van der Berg, S. (2008). Poverty and education. Education policy series, 10, 1-35. https://citeseerx.ist.psu.edu/viewdoc/download?doi=10.1.1.464.9607\&rep=rep1\&type=pdf 